\title{
Pulmonary consolidation and inflammation: inflammatory pseudotumour in young children
}

\author{
C.A. Lindemans1, R.A. Bruinsma2, P.G.J. Nikkels³, \\ F.H.L. Bröker4, H.G.M. Arets2
}

ABSTRACT: Pulmonary consolidation and inflammation: inflammatory pseudotumour in young children. C.A. Lindemans, R.A. Bruinsma, P.G.J. Nikkels, F.H.L. Bröker, H.G.M. Arets.

Here we report two children with a pulmonary inflammatory pseudotumour; a rare entity in children, that often initially presents as a pneumonia, but with the possibility of serious consequences if unrecognised and un- treated. One of the children presented is 6 months which is extremely young for this tumour. Difficulties in presentation, management strategies and prognosis are described. Certainly, this is a condition that should be considered even in a very young child with an inflammatory condition presenting as a solid lesion in the lung which does not resolve or even progresses.

Monaldi Arch Chest Dis 2009; 71: 2, 71-75.

Keywords: Infalmmatory pseudotumour, Myofibroblastic tumour, Inflammation, Neoplasm, Infants.

1 Department of Pediatrics, Gelre Hospitals, Lukas location,

2 Department of Pediatric Pulmonary Diseases, Wilhelmina Children's Hospital, University Medical Center, Utrecht,

3 Department of Pathology, University Medical Center, Utrecht,

4 Department of Radiology, Gelre Hospitals, Lukas location, Apeldoorn, the Netherlands.

Correspondence: Dr. C.A. Lindemans, Wilhelmina Children's Hospital, KE 04.133.1, P.O. Box 85090, 3508AB Utrecht, The Netherlands; e-mail: c.a.lindemans@umcutrecht.nl

Primary lung tumours are rare in children; with the exclusion of cystic lesions, they have been found to be malignant in the majority of cases [1]. The most common "benign" primary lung tumour in pediatric patients has been described to be the inflammatory pseudotumour, also known as inflammatory myofibroblastic tumour or plasma cell granuloma $[2,3]$. The occurrence of pseudotumours is not confined to the lung but can develop in every organ, with the lung, orbit and abdominal mesentery being the most common tumour sites $[4,5]$. The exact incidence of the pulmonary variant is unknown due to the diverse nomenclature that has been used to describe this condition, but lies somewhere between 0.04 to $0.7 \%$. The peak prevalence of pulmonary pseudotumours is the second decade of life and inflammatory pseudotumours have rarely been reported in infants. Here we report two children with a pulmonary inflammatory pseudotumour; a rare condition in children, that often initially presents as a pneumonia, but with the possibility of serious consequences if unrecognised and untreated.

\section{Case 1}

A 6 month old boy was referred to our regional clinic by the general practitioner, with fever and cough, unresponsive to broad spectrum antibiotic treatment. He had been treated with antibiotics for 7 days, prescribed by the general practitioner under the clinical suspicion of a bacterial pulmonary infection with high grade fever. On hospital presentation, he showed tachypnoea with a respiratory rate of $60 / \mathrm{min}$, difficult breathing and nasal flaring. Ausculation of the chest was unremarkable and he was diagnosed as having a viral lower respiratory tract infection.

The patient had a medical history of premature birth, being the second of twins born after 35 6/7 weeks gestation, birth weight 1850 grams. There had been no perinatal problems and he demonstrated catch-up growth (weight $0 \mathrm{SD}$; length -1SD).

When the boy's fever persisted and his condition worsened he was admitted to hospital. He was mildly dyspnoeic and ausculation and percussion of the chest were again normal. Surprisingly, a chest X-ray (fig. 1A ) demonstrated a consolidation of the left upper lobe. A blood specimen showed leucocytosis $(29.6 / \mathrm{nl})$, mainly polymorphous, trombocytosis $(727 / \mathrm{nl})$ and anaemia $(5.6$ $\mathrm{mmol} / \mathrm{l}$ ). C-reactive protein was $188 \mathrm{mg} / \mathrm{l}$ and erythrocyte sedimentation rate $95 \mathrm{~mm} / \mathrm{hr}$. After being diagnosed as having a pneumonia, he was treated with antibiotics (amoxilline/clavulanic acid), but with insufficient improvement. Two weeks later, he returned with the same symptoms and no improvement of either infection parameters or chest 


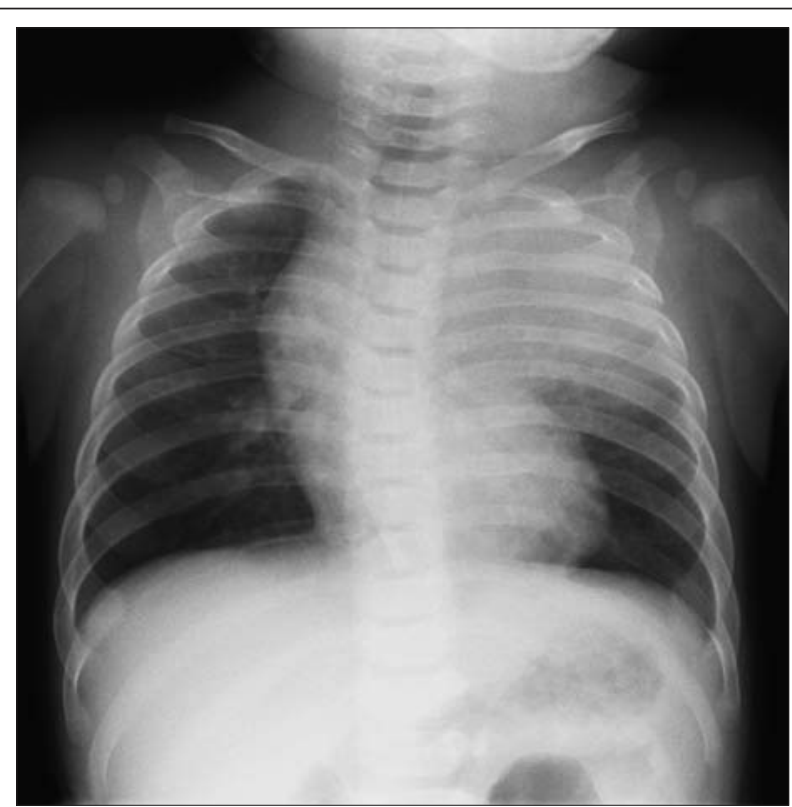

Fig. 1A. - The frontal view of the chest shows an homogeneous density involving the left upper lobe, suggestive of a lobar infiltrate. There is some space occupying effect with mediastinal shift to the right, causing a pronounced contour of the right thymic lobe. There are no signs of hilar or mediastinal lymphadenopathy.

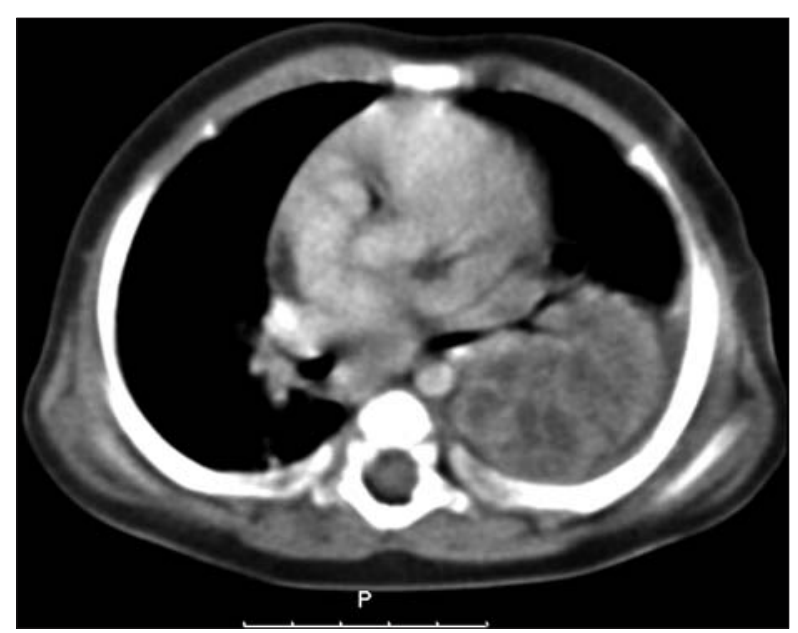

Fig. 1B. - A contrast-enhanced CT-image of the chest at the level of the left hilum demonstrates a lobulated and rather sharply demarcated dense mass of $4.5 \mathrm{~cm}$ diameter with irregular peripheral contrast enhancement involving the left upper lobe. There is a small amount of pleural effusion. No calcifications are seen.

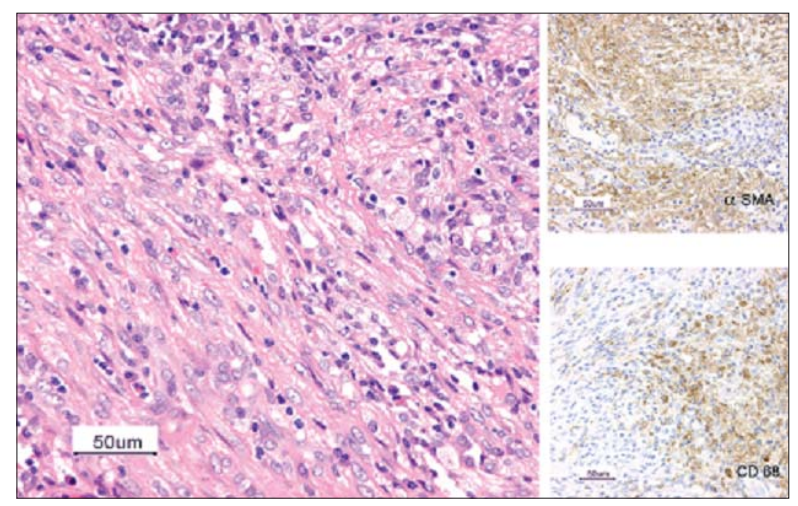

Fig. 1C. - Histology of the tumour showed highly proliferative myofibroblastic spindle cells arranged in bundels. In between bundles, are both foam cell macrophages and mononuclear cells (lymphocytes an plasma cells) and a small number of eosinophils. In more detail on the right: spindle cells were found to be $\alpha$-SMA positive and the foam cell macrophages were CD68 positive.
X-ray abnormalities. Chest ultrasound now showed consolidation of the left upper lobe with pleural effusion. A contrast-enhanced spiral CTscan of the chest (fig. 1B) showed a solid lesion in the left upper lobe with a diameter of $4.5 \mathrm{~cm}$. Under the suspicion of an abcess the boy was refered to a university hospital for surgical drainage. The process was found to be purulent peroperatively, and a left upper lobectomy was performed. Histology of the lesion showed highly proliferative myofibroblastic spindle cells with an infiltrate of mononuclear cells and foam cell macrophages (fig. 1C). Sporadically, necrotic debris was found, with clusters of neutrophils. Histology findings were in line with the diagnosis of an inflammatory pseudotumour. The post-operative period was mildly complicated by bronchospasms which were responsive to nebulised salbutamol/ ipratropiumbromide, and temporary hyponatremia based on the syndrome of inappropriate antidiuretic hormone secretion. After surgery the fever disappeared within two days and C-reactive protein decreased to the level of $27 \mathrm{mg} / \mathrm{l}$ within a week. After a week of amoxicillin/clavulanic acid in the postoperative phase, he was kept on co-trimoxazol prophylaxis for three months to prevent infection in healing lung tissue.

After three years of follow-up he is in good condition with infrequent symptoms of bronchial hyperreactivity. His growth remained stable from two months after surgery on the -2 SD for length, while weight for length is on the $0 \mathrm{SD}$.

\section{Case 2}

A 6 year old girl (born in Turkey), presented with dyspnoea, fever and excessive perspiration. She was referred because of persistent chest X-ray abnormalities after treatment with antibiotics. There was atelectasis of the right lower lobe with deviation of the mediastinum and trachea to the right (fig. 2A).

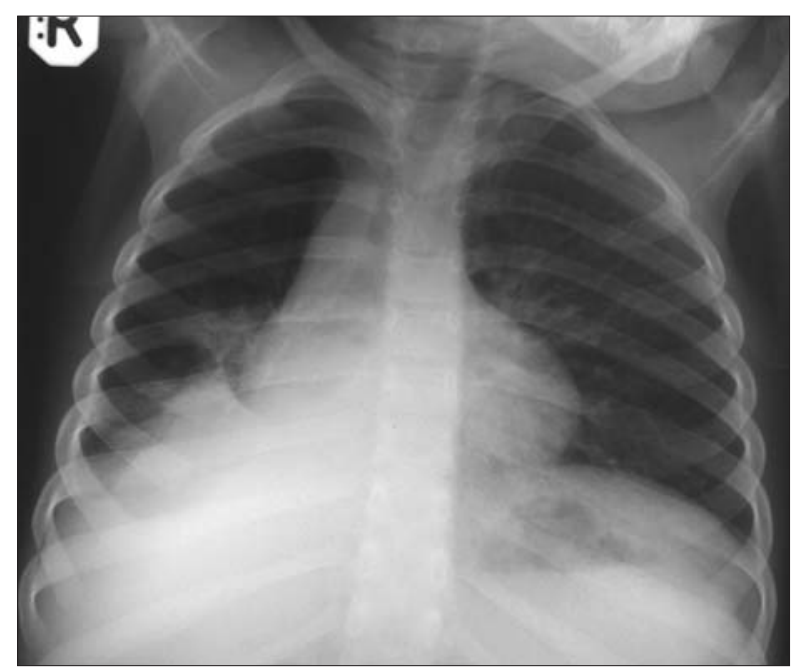

Fig. 2A. - The antero-posterior view of the chest on admission shows a sharply demarcated tent-shaped configuration in the right lower field in continuity with a right-sided retrocardial hyperdensity. The right hemidiaphragm is obscured by this consolidation. 


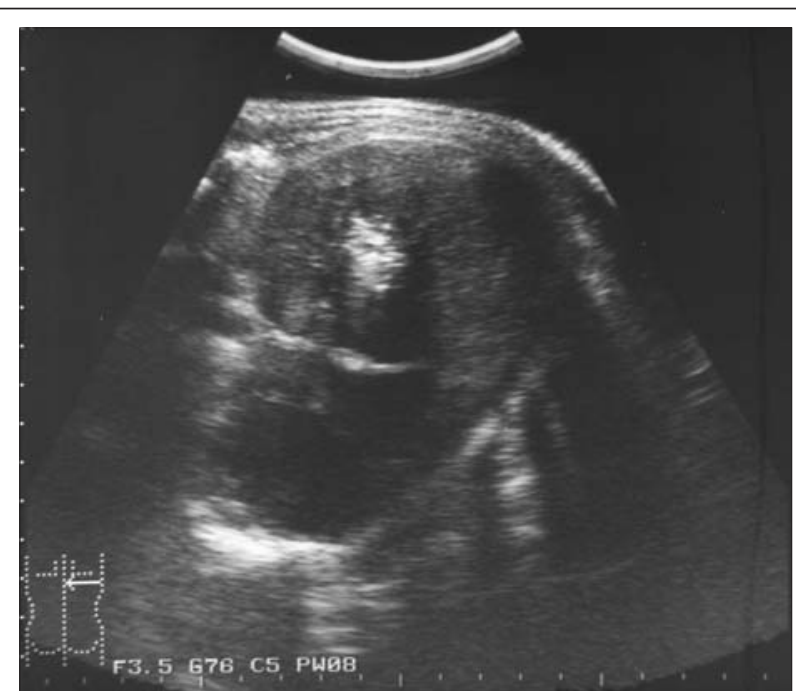

Fig. 2B. - A transversal ultrasound image from the dorsal side of the right lower chest demonstrates the presence of a round solid mass, in the posterior part of the right lower lobe, which measures $5 \mathrm{~cm}$ in diameter. It contains gross central calcifications.

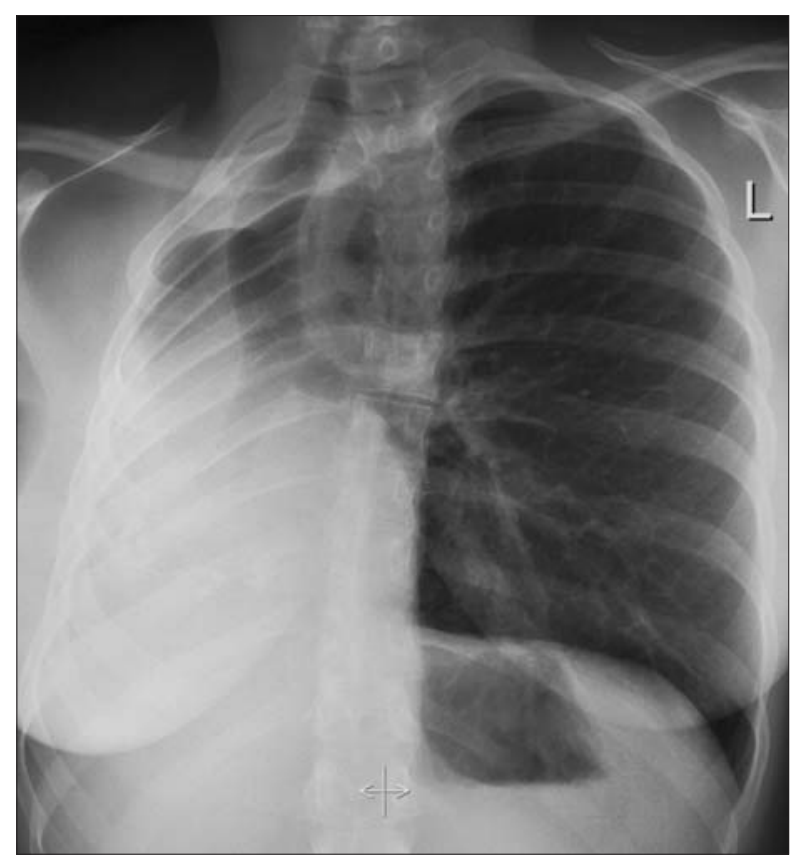

Fig. 2C. - The latest chest X-ray at the age of 21 years shows a completely collapsed or atelectatic right lung with considerable mediastinal shift to the right and compensatory hyperinflation of the left lung.

The family had moved to the Netherlands one month before her illness. She had a history of two episodes of pneumonia at the age of 3 and 4 years old respectively. Her father had suffered from tuberculosis, but our patient had been BCG vaccinated in Turkey.

There were no abnormalities on clinical examination. However, her length was $5 \mathrm{~cm}$ below -2 SD (corrected for Turkish orgin) and her weight for length at $+0.5 \mathrm{SD}$. Lab results showed an elevated erythrocyte sedimentation rate of $68 \mathrm{~mm} / \mathrm{hr}$, a normal leukocyte count $9.1 / \mathrm{hl}$, mild anaemia $(6.9 \mathrm{mmol} / \mathrm{l})$ and thrombocytosis $(524 / \mathrm{nl})$.

Chest ultrasound (fig. 2B) revealed the presence of a solid lobed process in the right lower lobe with a diameter of $5 \mathrm{~cm}$ and central calcification.
Repeated ultrasounds within a few weeks showed progression of this lesion. Bronchoscopy was performed and showed no foreign body but granulomatous tissue at the end of the pinpoint stenosed right main-stem and upper lobe bronchus, from which a biopsy was taken. Treatment for tuberculosis was initiated, but when this diagnosis wasn't confirmed by biopsy specimens, the treatment was discontinued. A second percutaneous ultrasound guided biopsy was taken from the mass in the right upper lobe and the histology showed solid areas of proliferating fibroblast with collagen and fibrosis with a mixed inflammatory infiltrate. These pathology characteristics were consistent with the diagnosis of an inflammatory pseudotumour. Awaiting diagnosis her condition had further deteriorated with atelectasis of the whole right lung. Because of extensive involvement of central airway and vascular structures, a non-surgical management was decided upon. She was treated with $20 \mathrm{mg}$ of cyclophosphamide daily for 6 weeks and oral steroids (prednisone $60 \mathrm{mg} / \mathrm{m}^{2}$ ) for 4 months and within weeks after initiation of this aggressive medical treatment, fever subsided and inflammatory parameters normalised. Because inflammatory pseudotumour is known for its risk of recurrence and malignant transformation, her condition has been monitored closely. Several episodes of bacterial pneumonia urged prophylactic treatment with oral amoxicillin/clavulanic acid for one year, but the inflammatory pseudotumour hasn't recurred. The patient is now 21 years old and so far, her condition has remained stable over the many years of followup, with persistent atelectasis, compensatory expansion of the left lung (fig. 2C) resulting in normal static lung volumes (TLC 3.07 liter $=87 \%$, RV $0.92=97 \%$, VC 2.15 liter $=80 \%$ ) and with relatively mild symptoms of bronchusobstruction $\left(\mathrm{FEV}_{1} 1.82\right.$ liter $\left.=77 \%\right)$ which are unresponsive to bronchodilator medication.

\section{Discussion}

The first case, reports one of the youngest patients so far described with a pulmonary inflammatory pseudotumour. Although inflammatory pseudotumours have been described in children, until now, no infants with pulmonary localization of this condition have been reported. In contrast to this, there have been a few literature reports of very young infants with a cardiac inflammatory pseudotumour. While the cardiac site may be rare, a rapidly growing mass localised in the heart will lead to symptoms associated with in- or out-flow obstruction and subsequently to an early diagnosis [5-7]. Of all the patients described with a pulmonary inflammatory pseudotumour, 30-70\% were asymptomatic [8, 9].

In general, respiratory symptoms are the most common presenting symptoms in children visiting a physician, especially when they present in combination with fever. In both of our patients, although children of completely different ages, the patients were initially diagnosed with a bacterial lower respiratory tract infection. The clinical pic- 
ture associated with the disease is that of inflammatory mediator release with fever, growth impairment, increased sedimentation rate and acute phase reactants irrespective of the localisation. In addition to that, the tumour can induce induce specific symptoms in specific organs such as out-flow obstruction of the airway or heart.

The possibility of a tumour in a child is considered only when radiographic abnormalities persist and as a complicating factor, radiologically, these tumours cannot be discriminated from other benign or malignant conditions [2]. The first patient showed consolidation in the upper lobe while the other showed consolidation and progressive atelectasis of the middle and lower lobe. Atelectasis is an uncommon presentation of pulmonary inflammatory pseudotumours in children [10]. In this case it has been explained by constriction of scarred tissue rather than by compression. Most importantly for establishing the diagnosis, both clinical symptoms and radiologic abnormalities proved to be refractory to antibiotic therapy. In adult patients a primary lung tumour would then be a very probable diagnosis. In contrast to adults however, neoplasms of the lung in pediatric patients are extremely rare and respiratory symptoms presenting with fever aremost likely to be of infectious origin. Tuberculosis was excluded in both cases. In the second patient who was of Turkish origin, fungi, Histoplasma and Echinococcus were also investigated as possible causative agents. In addition, Bartonella Henselae could be considered as an infectious agent regardless of ethnic origin $[11,12]$.

When radiological abnormalities with an unexplained dense lesion and/or fever do not respond to antibiotic treatment, biopsy or surgical resection of the lesion for pathology should be performed for both diagnostic and therapeutic reasons $[8,9,13$, 14]. No other investigation will be helpful in making the diagnosis of an inflammatory pseudotumour, as is recorded by several independent authors [15-18].

Histologically, the tumour-like lesion consists of myofibroblastic spindle cells and a heterogeneous inflammatory cell infiltrate of both $\mathrm{T}$ and $\mathrm{B}$ cells $[5,15]$. This cell heterogeneity discriminates the tumour from a lymphoma. Although it has these benign characteristics, pseudotumours have the capacity to be locally aggressive even with progression to adjacent organs, to recur and to become multifocal and occasionally malignant $[13$, $16,17]$. Despite their "benign" nomenclature, inflammatory pseudotumours should be considered to be true neoplasms as they do show clonal chromosomal abberations and aneuploidy on a molecular level [18-20].

There is an association of development of these pseudotumours secondary to infection by Mycoplasma, EBV or HHV-8 [5]. Previously, it was suggested that inflammatory pseudotumour could arise as a reactive process in response to either an infectious agent or a traumatic incident. However, only $20 \%$ of patients have clearly documented preceding infection or trauma $[2,21]$.
In the two cases described here, one patient was treated by surgical intervention, the other was treated with prednisone and cyclophosphamide. Both children had a favourable outcome. In adults, the treatment of choice is radical surgical resection $[9,13,22]$. The main reason for this is that the chance of local recurrence is $25 \%$ and that there is a small chance of malignancy $[14,23]$. The second treatment of choice is corticosteroids with $50 \%$ of tumour responsiveness [5, 24]. As a last resort, or as adjuvant therapy, other immunosuppressive chemotherapeutics (such as MTX and cyclophosphamide) and irradiation can be attempted to induce remission. For pseudotumours in close localisationto vital structures such as orbital pseudotumours, corticosteroids are the treatment of choice [25]. In steroid resistent tumours, irradation has a proven, but limited effect as have other immunosuppressive agents such as cyclophosphamide, ciclosporine and MTX $[5,25,26]$. Consequently, after treatment with immunosuppressants such as in the second case described with a relative contraindication for major surgery, a long and thorough follow-up is required to monitor recurrent local invasion, the development of distant metastasis and malignant transformation.

In conclusion, inflammatory pseudotumours exhibit a benign nature with an excellent chance of complete resolution after surgical resection. However, the malignant characteristics described above, disclose their neoplastic potential. Certainly, this is a condition that should be considered even in a very young child with an inflammatory condition presenting as a solid lesion in the lung which does not resolve or even progresses.

Conflict of Interest Statement: There are no financial or personal relationships with others persons or organisations that could have biased this paper. We would like to inform you that case 2 has been published before in another form and for another audience in 1999 [10]. Figure 2A, B and C have however never been published and the case was added because of the contrast in management between the two.

\section{References}

1. Hancock BJ, Di LM, Youssef S, Yazbeck S, Marcotte JE, Collin PP. Childhood primary pulmonary neoplasms. J Pediatr Surg 1993; 28: 1133-1136.

2. McCahon E. Lung tumours in children. Paediatr Respir Rev 2006; 7: 191-196.

3. Hartman GE, Shochat SJ. Primary pulmonary neoplasms of childhood: a review. Ann Thorac Surg 1983; 36: 108-119.

4. Coffin CM, Watterson J, Priest JR, Dehner LP. Extrapulmonary inflammatory myofibroblastic tumour (inflammatory pseudotumour). A clinicopathologic and immunohistochemical study of 84 cases. Am J Surg Pathol 1995; 19: 859-872.

5. Narla LD, Newman B, Spottswood SS, Narla S, Kolli R. Inflammatory pseudotumour. Radiographics 2003; 23: 719-729.

6. Hartyanszky IL, Kadar K, Hubay M. Rapid recurrence of an inflammatory myofibroblastic tumour in the right ventricular outflow tract. Cardiol Young 2000; 10: 271-274.

7. Li L, Cerilli LA, Wick MR. Inflammatory pseudotumour (myofibroblastic tumour) of the heart. Ann Diagn Pathol 2002; 6: 116-121. 
8. Berardi RS, Lee SS, Chen HP, Stines GJ. Inflammatory pseudotumours of the lung. Surg Gynecol Obstet 1983; 156: 89-96.

9. Cerfolio RJ, Allen MS, Nascimento AG, et al. Inflammatory pseudotumours of the lung. Ann Thorac Surg 1999; 67: 933-936.

10. Verbeke JI, Verberne AA, Den Hollander JC, Robben SG. Inflammatory myofibroblastic tumour of the lung manifesting as progressive atelectasis. Pediatr Radiol 1999; 29: 816-819.

11. Marseglia GL, Monafo V, Marone P, Meloni F, Martini A, Burgio GR. Asymptomatic persistent pulmonary infiltrates in an immunocompetent boy with cat-scratch disease. Eur J Pediatr 2001; 160: 260-261.

12. Caniza MA, Granger DL, Wilson KH, et al. Bartonella henselae: etiology of pulmonary nodules in a patient with depressed cell-mediated immunity. Clin Infect Dis 1995; 20: 1505-1511.

13. Kovach SJ, Fischer AC, Katzman PJ, et al. Inflammatory myofibroblastic tumours. J Surg Oncol 2006; 94: 385-391.

14. Melloni G, Carretta A, Ciriaco P, et al. Inflammatory pseudotumour of the lung in adults. Ann Thorac Surg 2005; 79: 426-432.

15. Matsubara O, Tan-Liu NS, Kenney RM, Mark EJ. Inflammatory pseudotumours of the lung: progression from organizing pneumonia to fibrous histiocytoma or to plasma cell granuloma in 32 cases. Hum Pathol 1988; 19: 807-814.

16. Janik JS, Janik JP, Lovell MA, Hendrickson RJ, Bensard DD, Greffe BS. Recurrent inflammatory pseudotumours in children. J Pediatr Surg 2003; 38: 1491-1495.

17. Kato S, Kondo K, Teramoto T, et al. A case report of inflammatory pseudotumour of the lung: rapid recurrence appearing as multiple lung nodules. Ann Thorac Cardiovasc Surg 2002; 8: 224-227.
18. Chun YS, Wang L, Nascimento AG, Moir CR, Rodeberg DA. Pediatric inflammatory myofibroblastic tumour: anaplastic lymphoma kinase (ALK) expression and prognosis. Pediatr Blood Cancer 2005; 45: 796801.

19. Su LD, Tayde-Perez A, Sheldon S, Fletcher JA, Weiss SW. Inflammatory myofibroblastic tumour: cytogenetic evidence supporting clonal origin. Mod Pathol 1998; 11: 364-368.

20. Biselli R, Ferlini C, Fattorossi A, Boldrini R, Bosman C. Inflammatory myofibroblastic tumour (inflammatory pseudotumour): DNA flow cytometric analysis of nine pediatric cases. Cancer 1996; 77: 778-784.

21. Dahabreh J, Zisis C, Arnogiannaki N, Katis K. Inflammatory pseudotumour: a controversial entity. Eur $J$ Cardiothorac Surg 1999; 16: 670-673.

22. Messineo A, Mognato G, D'Amore ES, Antoniello L, Guglielmi M, Cecchetto G. Inflammatory pseudotumours of the lung in children: conservative or aggressive approach? Med Pediatr Oncol 1998; 31: 100-104.

23. Morotti RA, Legman MD, Kerkar N, Pawel BR, Sanger WG, Coffin CM. Pediatric inflammatory myofibroblastic tumour with late metastasis to the lung: case report and review of the literature. Pediatr Dev Pathol 2005; 8: 224-229.

24. Doski JJ, Priebe CJ, Jr., Driessnack M, Smith T, Kane $\mathrm{P}$, Romero J. Corticosteroids in the management of unresected plasma cell granuloma (inflammatory pseudotumour) of the lung. J Pediatr Surg 1991; 26: 10641066.

25. Jacobs D, Galetta S. Diagnosis and management of orbital pseudotumour. Curr Opin Ophthalmol 2002; 13: 347-351.

26. Diaz-Llopis M, Menezo JL. Idiopathic inflammatory orbital pseudotumour and low-dose cyclosporine. Am J Ophthalmol 1989; 107: 547-548.

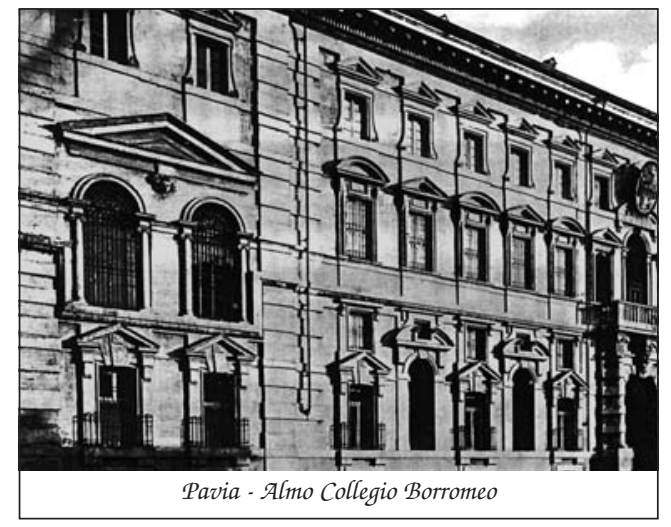

